\title{
THE INFLUENCE OF BORDER MOLDING ON RETENTION AND TIME OF COMPLETE DENTURE IMPRESSION
}

\author{
Amr A Rady* and Nouran A El Naby*
}

\begin{abstract}
Background: Maintenance of contact of the denture border with the adjacent vestibular tissues during rest as well as in function is an important criterion of denture success that depends on proper border tracing. Different border moulding materials are available for achievement of that goal although many clinicians deliver excellent denture without border trimming procedure. The aim of this study is firstly to evaluate the time needed to perform sectional border molding with two different border tracing materials and to compare the retention of the finished impressions with non-border molded impression.
\end{abstract}

Material and method: Twelve completely edentulous patients were selected and signed consents to evaluate the retention provided by three different final impression techniques (sectional border moulding of a special tray with low fusing compound and final wash with medium bodied silicone material, sectional border tracing of a special tray with putty elastomeric impression material followed by a final wash with medium bodied silicone material and third impression with special tray without border moulding). A stop watch was used to record the time needed to finish the border moulding in the first two impression technique, then a digital force meter was used to measure retention strength provided by each technique.

Results: The statistical analysis showed extremely significant difference between the time needed to finish border moulding with putty silicone material and that with the low fusing compound. Moreover, no significant difference was detected between the three impressions techniques.

Conclusion: Putty silicone material allows faster, easier and effective border moulding procedure rather than low fusing compound. Medium body silicone material in special tray without border moulding yielded highly retentive impression comparable to those with different border tracing materials.

\section{INTRODUCTION}

Border molding is an important process in complete denture construction as it promotes the development of border seal which is necessary for the maintenance of contact of the denture border with the adjacent vestibular tissues during rest as well as in functional activity. Border trimming is defined as the shaping of an appropriate impression

* Lecturer of Removable Prosthodontics, Faculty of Oral and Dental Medicine, Cairo University. 
material by action of the tissues adjacent to the borders of an impression tray. Kaur S et al 2016 conducted a study on importance of border molding and proved that the dentures made with border molding will provide better retentive force than the dentures made without border molding ${ }^{(1)}$

Peripheral seal is established when denture borders contact with the underlying or adjacent tissues and prevent passage of air or other substances. It is one of the important factors that will provide optimal retention of the denture promoting psychologic comfort and patient acceptance to the finished prosthesis ${ }^{(2)}$.

Ideally, The molding material should possess certain criteria such as sufficient body to allow it to remain in position on the border during loading of the tray, homogenous consistency to offer uniform resistance to displacement by the vestibular tissues and adequate flow for sufficient time to be functionally molded by tissue ${ }^{(3)}$

Impression modeling plastic has been always used for border molding complete denture impression trays using a sectional technique where parts of the borders are molded in separate applications. Peripheral areas can be molded with less distortion or breakage of the completed sections; moreover, corrections or additions to the earlier molded segments can be easily accomplished rendering the modeling plastic to be the material of choice for teaching ${ }^{(4)}$.

Since there is no support for the frequent textbook statement that the sectional procedure is necessary and superior to the one-step method, materials which allow simultaneous molding of all borders in a one-step technique have gained more preference by many clinicians, saving great time due to reduced number of tray insertions, causing less discomfort for the patient and avoiding propagation of errors caused by mistake in one section affecting the border contour in another section ${ }^{(5)}$.
Elastomeric impression materials are most commonly used as a substitute for low fusing compound as they fulfill most of the requirements. The introduction of elastomeric impression materials $^{(6)}$ has made possible new techniques of recording impression for complete denture construction. Heavy body putty silicone has been used for border molding instead of low fusing compound. It can be placed continuously along the entire border of an individual tray, and the border of the tray can be molded at a single stage. In addition, it also had high degree of accuracy, dimensional stability and ease of manipulation ${ }^{(6,7)}$.

\section{Aim of study}

The purpose of this study is firstly to evaluate the time needed to perform sectional and single step border molding and to compare the retention of the finished impressions with non-border molded impression.

\section{MATERIAL AND METHOD}

Informed consents were signed by twelve patients who showed interest to share in this study after making sure of their freedom from any inflammation affecting the residual alveolar ridges. Upper preliminary impression was taken in alginate; diagnostic cast was made and undercuts blocked out with baseplate wax. One autopolymerised resin custom tray (Major Tray Mmajor Prodotti Dentari Spa, Moncalieri, Torino, Italy) was constructed over diagnostic cast, checked and adjusted intraorally. A metal loop is attached to the geometric center of the tissue away surface of the tray at the point of intersection of canine-tuberosity lines (Fig.1).

\section{Impression procedures}

Green stick compound (Kerr Italia S.P.A. 1-84014 Scafti, Salerno-Italy) was glazed over the flame, applied to the tuberosity region of the tray then tempered in hot water before insertion in the patient's mouth and the patient was asked to pro- 
trude and move the mandible from side to side. The posterior palatal seal was functionally performed by applying the green stick compound at the posterior border of the tray from one hamular notch to the other and asking the patient to swallow. Buccal and labial tracing of the tray was carried out by adding soft compound section by section to the flanges and moving the cheeks and lips upwards forwards and downwards. Any excess compound flowing onto the fitting surface of the tray was scrapped using sharp knife, a stop watch was used to record the time needed to finish the molding until the tracing was satisfactory (Fig.2).

Tray adhesive (Virtual Tray Adhesive, Ivoclar Vivadent, Schaan, Liechtenstein) was applied to internal aspect of the tray, borders of the tray were reduced by 1-2 $\mathrm{mm}$ to allow space for the final wash impression using monophase silicone material (Virtual Monophase, Medium viscosity, Ivoclar Vivadent, Schaan, Liechtenstein). The tray and the cut down borders were coated with the impression material, seated lightly into mouth and the patient was asked to perform functional movements such as puckering the lips swallowing, speaking, smiling and move the mandible from side to side until the material set (Fig. 3).

The hook of the digital force meter (Holdwell Digital Push Pull Gauge Gage HF-500N Force Gauge Tester Meter, Hanghou Holdwell Mechanical \& Electical CO.,LTD, China) was suspended to the wire loop attached to the tissue away surface of the tray, a vertical downward force was applied to dislodge impression tray while the patient was sitting in an upright position with the occlusal plane parallel to the floor (Fig. 4). Three readings were recorded and the average was calculated.

Impression and border molding were removed from the tray, adhesive was applied over the borders, internal and external surface of the acrylic custom tray to facilitate the retention of the silicone border molding material. - Putty silicone

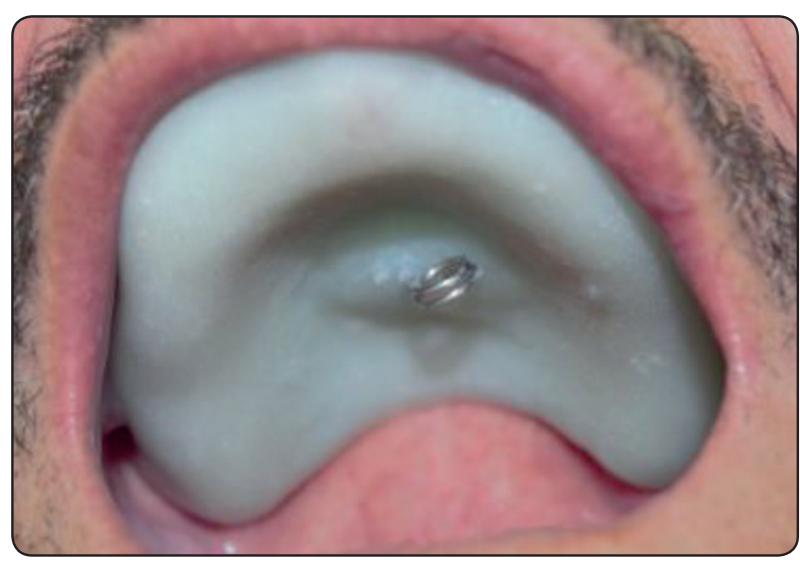

Fig. (1) Metal loop attached to the tray

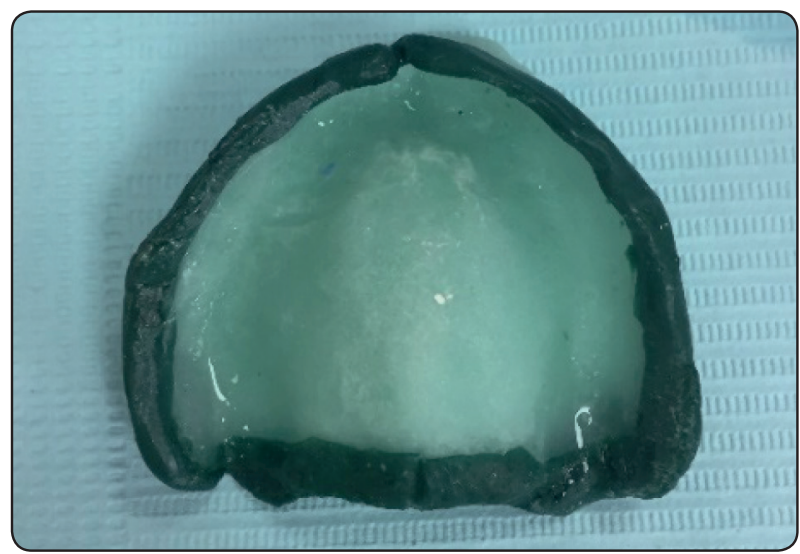

Fig. (2) Low fusing compound used for border moulding

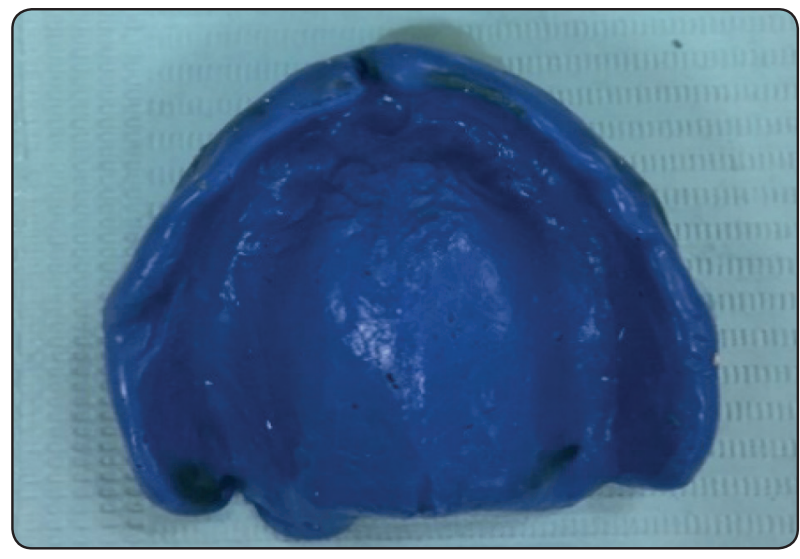

Fig. (3) Final impression after border moulding with low fusing compound 
(Virtual putty regular set, Ivoclar Vivadent, Schaan, Liechtenstein) was kneaded and a roll of 3-4 mm width was placed along the entire labial and buccal peripheries of the maxillary tray, followed by another insertion for posterior palatal border (Fig. 5), Patients were instructed to relax and perform the normal functional movements as described above. The border molding was examined for rounded contour and any deficient area is corrected with a small addition of putty material. New timing was recorded using the stopwatch before reducing the borders by 1 $\mathrm{mm}$ in preparation for the final impression that was taken using the monophase silicone material (Fig. 6) and force needed to displace the tray of the final impression was determined for another time using the digital force meter.

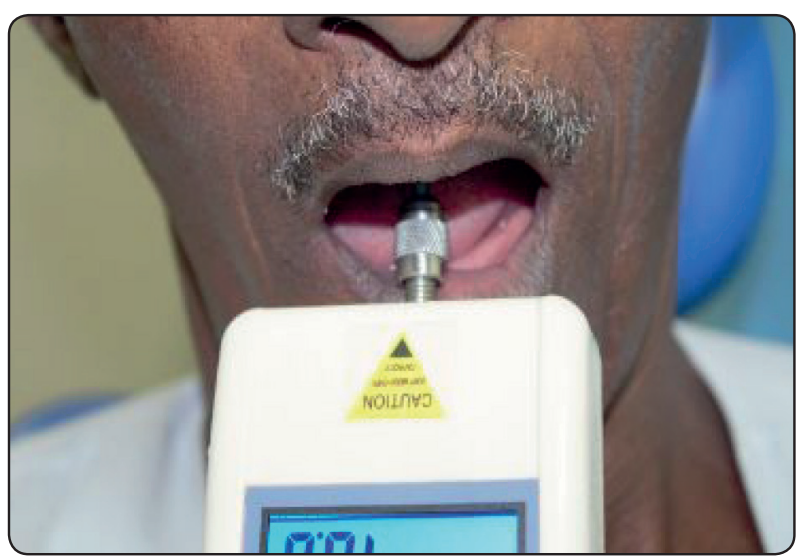

Fig. (4) Force meter used for recording retention

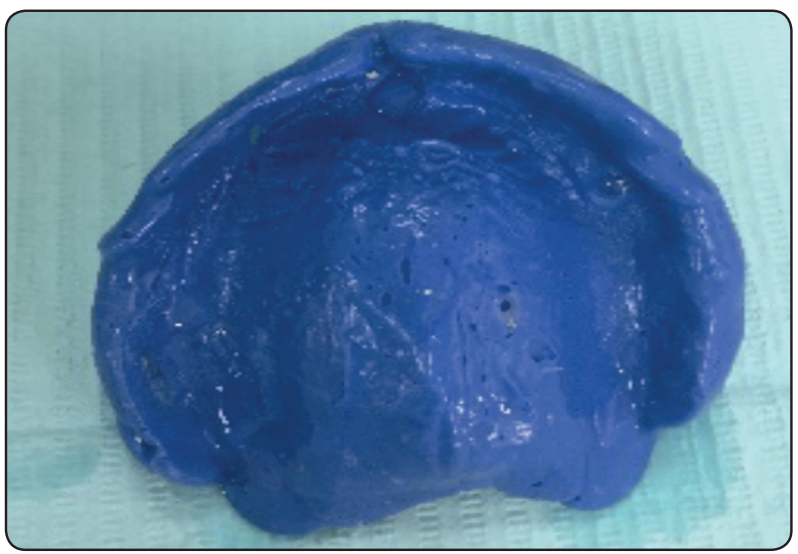

Fig. (6) Heavy bodied putty silicone used for border moulding
Finally, the impression and putty were removed and a third impression was made using the monophase silicone material alone after coating the tray with adhesive (Fig. 6) and final record of force needed to displace the tray was made using the force meter.

Results were shown as mean \pm SEM (standard error of means). Statistical significance between individual comparisons was determined using Student t-test. For multiple comparisons, oneway ANOVA with Tukey (comparisons between all groups) and Sidak's (selected pairs) pairwise tests were used. The calculations were performed with the statistical software package GraphPad Prism (version 7). P values $\leq 0.05$ were considered statistically significant.

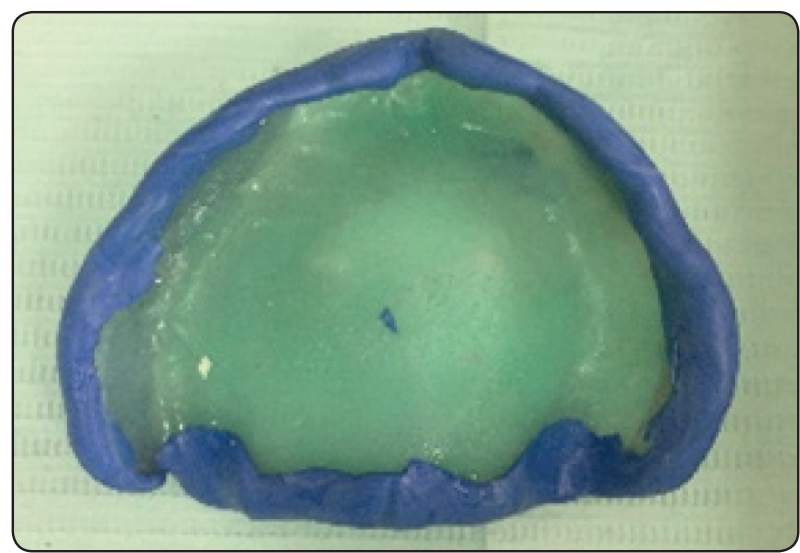

Fig. (5) Final impression after border tracing with putty

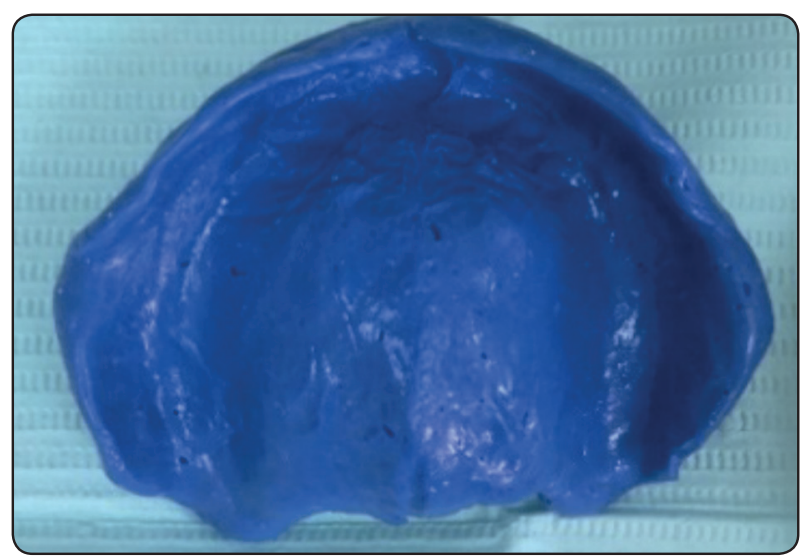

Fig. (7) Third final impression without border moulding material 


\section{RESULTS}

In the current research, time needed to finish the border tracing using two different moulding materials was recorded for all patients included in the study and Two-tailed unpaired t test showed an extremely statistical significant difference $(\mathrm{p}<0.0001)$ between the two groups. The means of time for border moulding materials were 2.31 minutes for putty rubber base and 5.39 minutes for low fusing compound.

\begin{tabular}{|l|l|}
\hline Two-tailed unpaired $\mathrm{t}$ test & \\
\hline P value & $<0.0001$ \\
\hline P value summary & $* * * *$ \\
\hline
\end{tabular}

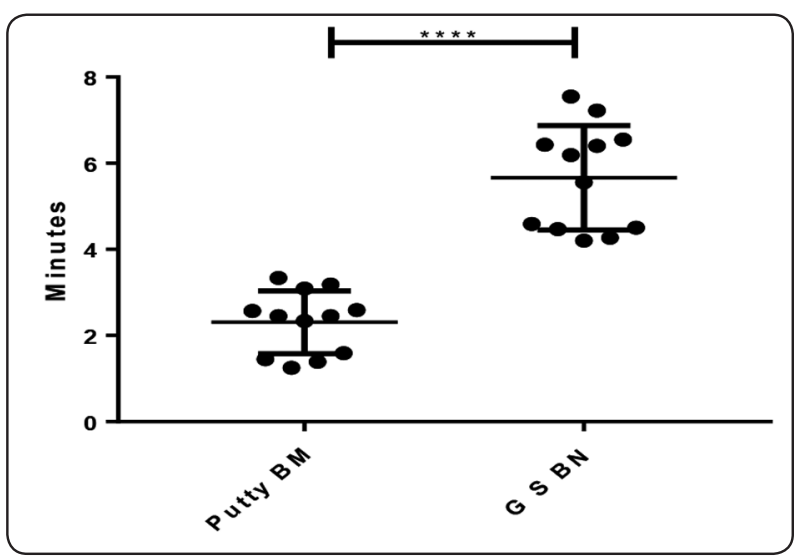

Fig. (8) Scatter blot showing extremely significant increase in the mean time in minutes of green stick compound border molding (GS BN when compared to the putty border molding (Putty border molding)

The three impression techniques were performed on all patients included in the study and ANOVA showed a non-statistical difference $(p=0.9864)$ in the overall retention force recorded for each technique where the means for of impression techniques were 63.65 for border moulding with low fusing compound with final wash of medium bodied silicone material, 62.125 for border moulding with putty silicone material with final wash of medium bodied silicone material and 62.975 for direct impression with medium bodied silicone material without border moulding. Furthermore, Tukey multiple comparison test revealed a nonsignificant change in the measured retention forces between each technique investigated.

\begin{tabular}{|l|l|}
\hline ANOVA & \\
\hline P value & 0.9864 \\
\hline P value summary & ns \\
\hline
\end{tabular}

\begin{tabular}{|l|l|r|}
\hline Tukey's multiple comparisons test & Summary & P Value \\
\hline Imp1 vs. Imp 2 & ns & 0.9851 \\
\hline Imp1 vs. Imp3 & ns & 0.9971 \\
\hline Imp 2 vs. Imp3 & ns & 0.9954 \\
\hline
\end{tabular}

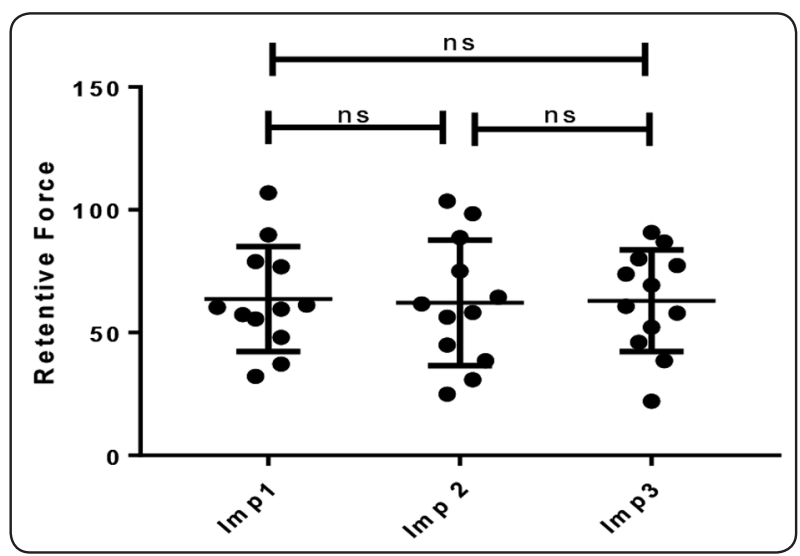

Fig. (9) Scatter Plot demonstrating the retention force of the three tested impressions. Dta represent the mean and SD of the retention force measured in Newtons (Retentive Force) and reveals a non significant difference(ns) among the tested impressions. $\mathrm{N}=12$ in each tested group. Imp1: low fusing compound border molding with medium body silicone impression, Imp2: putty silicone border molding with medium body silicone impression and Imp3: medium body silicone impression without border moding. 


\section{DISCUSSION}

A single acrylic resin custom impression tray was constructed for each patient to eliminate the possible variations that may arise from different trays adaptation and to obtain uniform bulk of the wash impression ${ }^{(8)}$. Moreover, the recording of retentive forces was done at the impression stage as in a previous study comparing the retention at the three stages border molding, final impression and permanent denture base steps, the result showed less retention at permanent denture base phase which may be attributed to technical errors like those involved in packing, curing or volumetric shrinkage of acrylic resin, resulting in improper fit of the permanent denture base with underlying soft tissue. Prior to measuring occlusal plane of each patient was adjusted parallel to the Frankfurt plane to ensure that forces are perpendicular on the tray ${ }^{(9)}$.

A loop was attached at the middle portion of the palatal surface which was the most consistent area for testing the retention of complete denture because the anterior attachment needed the greatest amount of force to displace the base in comparison to posterior region ${ }^{(10)}$.

The choice of materials in this study was low fusing compound for sectional border molding which is a gold standard in border tracing and addition silicone putty material for single step border molding because of its excellent manipulative consistency and dimensional stability. The final impression material used in the study was medium bodied addition silicone material as it was concluded that that dentures made from silicone impressions were preferred by patients ${ }^{(11)}$.

In the comparison of time between the two border molding materials, it was obvious and expected to find a statistically significant difference, where the time needed for the heavy bodied putty silicone material was significantly less than that for the low fusing compound. It has been reported that seven prosthodontic instructors required an average of 17 placements to obtain a maxillary final impression on the same patient using modeling plastic as the border molding material Added to that, low fusing compound has short manipulation time, hardens quickly in the mouth and does not remain in a plastic stage till the functional movements are completed thus requiring more insertions and consuming more time $^{(12)}$. In view of these limitations, putty silicone has been used for border molding where the labial and buccal borders can be recorded in one step and posterior palatal seal area in another step producing good results in less time as well as less discomfort and inconvenience for the patient ${ }^{(13)}$.This result is in accordance with patients opinions about the border molding procedure based on a previous study ${ }^{(14)}$.

Concerning retention evaluation, no statistically significant difference was detected between the three impression techniques with nearly equal means, indicating that heavy bodied putty silicone material and low fusing compound have similar effects as border molding materials. In accordance with ${ }^{(4,6)}$, trays traced with both materials required high forces to be displaced from their basal seats which will be reflected later on the final denture base retention. Moreover, the impression made without border molding yielded an acceptable retention comparable to the other impressions and this may be correlated to the fact that many operators around the globe used custom tray without border molding (15, 16) and this may be as well attributed to the medium viscosity of the monophase impression material that enable it to make up discrepancies between tray borders and the reflecting vestibular tissues of up to 4 or $5 \mathrm{~mm}^{(17,18)}$.

\section{CONCLUSION}

Based on the results of this study, it can be concluded that the heavy bodied putty silicone material allows faster sectional border molding than low fusing compound that requires more insertions for adjustment. Medium bodied addition silicone material produced high retentive values in completely edentulous maxillary impression when used with special tray for final impression with or without any border tracing material. 


\section{REFERENCES}

1. S. Kaur, K. Datta, S. K. Gupta, N. Suman, Comparative analysis of the retention of maxillary denture base with and without border molding using zinc oxide eugenol impression paste. Indian J Dent 7, 1-5 (2016).

2. T. E. Jacobson, A. J. Krol, A contemporary review of the factors involved in complete denture retention, stability, and support. Part I: retention. J Prosthet Dent 49, 5-15 (1983).

3. B. C. Zarb GA, Eckert SE, Prosthodontic treatment for edentulous patients. (Mosby, St.Louis, ed. 12th, 2003).

4. A. Qanungo et al., Comparative evaluation of border molding using two different techniques in maxillary edentulous arches: A clinical study. J Indian Prosthodont Soc 16, 340345 (2016).

5. G. E. Carlsson, A. Ortorp, R. Omar, What is the evidence base for the efficacies of different complete denture impression procedures? A critical review. J Dent 41, 17-23 (2013).

6. R. Yarapatineni et al., Comparative evaluation of border molding, using two different techniques in maxillary edentulous arches - An in vivo study. J Int Oral Health 5, 82-87 (2013).

7. A. K. Arora, I. Goyal, M. Sehgal, Comparative evaluation of reproducibility of peripheral tissues produced by different border molding materials in edentulous patients: An in vivo study. J Indian Prosthodont Soc 15, 102-110 (2015).

8. W. W. Chee, T. E. Donovan, Polyvinyl siloxane impression materials: a review of properties and techniques. J Prosthet Dent 68, 728-732 (1992).

9. R. Yarapatineni et al., Comparative evaluation of border molding, using two different techniques in maxillary eden- tulous arches - An in vivo study. Journal of International Oral Health : JIOH 5, 82-87 (2013).

10. A. Colon, K. Kotwal, A. D. Mangelsdorff, Analysis of the posterior palatal seal and the palatal form as related to the retention of complete dentures. J Prosthet Dent 47, 23-27 (1982).

11. T. P. Hyde et al., A randomised controlled trial of complete denture impression materials. J Dent 42, 895-901 (2014).

12. J. B. Woelfel, J. C. Hickey, T. Berg, Jr., Contour variations in one patient's impressions made by seven dentists. J Am Dent Assoc 67, 1-9 (1963).

13. E. M. Appelbaum, R. V. Mehra, Clinical evaluation of polyvinylsiloxane for complete denture impressions. J Prosthet Dent 52, 537-539 (1984).

14. R. Tasleem, M. H. Bin Saeed, M. U. Javed, Comparison of complete denture fabricated by two different border molding materials, in terms of patients' satisfaction. J Ayub Med Coll Abbottabad 25, 78-80 (2013).

15. E. E. Daou, The elastomers for complete denture impression: A review of the literature. The Saudi Dental Journal 22, 153-160 (2010)

16. T. D. Taylor, A. C. Matthews, S. A. Aquilino, N. S. Logan, Prosthodontic survey. Part I: Removable prosthodontic laboratory survey. J Prosthet Dent 52, 598-601 (1984).

17. H. K. Tan, P. M. Hooper, C. G. Baergen, Variability in the shape of maxillary vestibular impressions recorded with modeling plastic and a polyether impression material. Int $\mathrm{J}$ Prosthodont 9, 282-289 (1996).

18. D. A. Felton, L. F. Cooper, M. S. Scurria, Predictable impression procedures for complete dentures. Dent Clin North Am 40, 39-51 (1996). 